\title{
L'importance de la visibilité dans les processus migratoires
}

\section{Carmen Gómez Martín}

gomez.martin.carmen@gmail.com

\section{Résumé}

La question de la visibilité n’a été traitée, jusqu'à présent, que de manière marginale par la sociologie des migrations. Avec cet article nous allons de démontrer la pertinence de cette notion comme instrument analytique dans le domaine d'étude des migrations. Pour accomplir cet objectif, nous allons utiliser une nouvelle méthodologie, en incorporant la visibilité dans le cadre du processus plus ample et complexe de la reconnaissance. Nous appliquerons ce schéma de travail, par la suite, au domaine des migrations en Espagne à fin de démontrer la validité de notre démarche.

Mots clé: visibilité, sociologie des migrations, reconnaissance, Espagne.

Abstract. The importance of visibility in migratory processes

The Sociology of Migration has treated the «visibility» question in rather marginal way so far. With this paper we try to demonstrate the soundness of this concept as an analytical tool in migration studies. To this end, we will use a new methodology consisting in incorporating «visibility» to the wider and more complex «recognition» process. Finally, we will apply this approach to the migration question in Spain to further proof its validity.

Key words: visibility, migration studies, recognition process, Spain.

\section{Sommaire}

1. Introduction 4. La visibilité et ses implications

2. La distinction entre visibilité dans la migration

et reconnaissance Conclusion

3. En quête de visibilité. Le processus de la reconnaissance vu de l'intérieur

Références bibliographiques 


\section{Introduction ${ }^{1}$}

La notion de visibilité est un terme complexe qui suscite de nos jours un fort intérêt mais qui, en même temps, se caractérise par une importante imprécision théorique. Dans cet article nous souhaitons contribuer à la compréhension de cette notion à partir d'une nouvelle perspective d'étude, c'est-à-dire sa mise en relation avec le terme et le processus de la reconnaissance. Nous partons de l'idée que les deux notions, en étant différentes, font partie cependant, d'un même processus qui a été dénommé "processus de la reconnaissance»; processus qui peut être décomposé en plusieurs étapes, ou moments, dont la visibilité et la reconnaissance feraient partie de l'une d'entre elles.

Cette nouvelle construction théorique autour du concept de visibilité nous permet de problématiser notre objet d'étude, en le dotant d'une valeur comme outil analytique en sciences sociales. Cela nous amènera, dans un second temps, à nous pencher directement sur le champ des migrations. Nous allons utiliser comme cadre théorique de référence les théories historico-structurelles, et plus concrètement le modèle de réseaux sociaux, car il tient compte des facteurs non seulement économiques, mais aussi, sociaux, historiques et culturels des contextes d'émission et de réception pour expliquer les phénomènes migratoires. Par conséquent, on introduira la visibilité ici en tant que facteur servant à compléter les explications apportées par ce modèle. Notre instrument d'analyse agit sur un double plan. D'abord, il amène à comprendre comment et pourquoi se produit la formation des discours exclusion/inclusion qui influencent la construction des imaginaires collectifs sur «autrui»; et deuxièmement, à reconnaître les efforts développés par les propres immigrés pour contrôler leur image et leur propre destin, ainsi que pour être reconnus comme acteurs sociaux de premier ordre dans les sociétés d'accueil.

\section{La distinction entre visibilité et reconnaissance}

Le terme de visibilité a souvent été assimilé à d'autres concepts dont celui de reconnaissance, ce qui expliquerait, au moins partiellement, la faible élaboration théorique autour du premier concept. La confusion existante entre visibilité et reconnaissance vient du fait de leur coexistence dans un même processus, «le processus de la reconnaissance». La tradition de pensée qu'inaugure Charles Taylor au début des années 1990 définit l'importance capitale de ce processus dans l'évolution des sociétés contemporaines. Ce courant établit une étroite connexion entre le processus de reconnaissance et la formation de l'identité des sujets, c'est-à-dire que la formation de notre identité se trouve en étroite

1. Le présent article fait partie d'un travail de recherche plus large qui a comme objet d'étude la notion de visibilité et ses implications dans les processus migratoires. Le texte que nous allons présenter ici a été conçu afin d'exposer des aspects plus théoriques de notre recherche. Cependant, nous voudrions signaler que ce travail théorique s'appuie fortement sur un terrain d'application pratique, la migration des Kurdes et des Sahraouis dans le contexte plus général de l'immigration en Espagne, qui fera l'objet de prochains articles. 
connexion avec la reconnaissance sociale basée sur le respect et l'acceptation. La reconnaissance donc, selon Taylor (1992), n'est pas simplement le produit d'un travail introspectif sur nous-mêmes, mais aussi le résultat d'une négociation, d'un dialogue ouvert avec les autres.

La forte imbrication qui existe entre visibilité et reconnaissance dans ce processus facilite le glissement du premier terme vers le second. Cependant, si nous considérons le processus de la reconnaissance dans son ensemble, nous percevons qu'il n'est pas acquis une fois pour toute, mais plutôt qu'il est composé de plusieurs étapes ou moments, la visibilité étant une d'entre elles. Autrement dit, la reconnaissance ne serait que la phase achevée d'un processus qui a comme point de départ une demande de visibilité. Néanmoins, il faut tenir compte du fait que ce processus n'est pas toujours accompli dans sa totalité et avoir une visibilité ne veut pas dire que celle-ci engendrera nécessairement la reconnaissance d'un sujet ou d'un groupe.

La séparation entre les deux concepts a été soutenue par des auteurs tels qu'Olivier Voirol (2005) ou Axel Honneth (2006). En ce sens, ce dernier établit l'existence de deux moments qui configurent l'acte de la reconnaissance (Honneth, 2006: 46). D'un côté, il y aurait ce qu'il appelle l'«identification cognitive», c'est-à-dire quand un sujet est perçu par autrui en tant que tel avec des caractéristiques particulières. Dans un second temps, Honneth décrit une autre étape nommée l' «expression» où il existe une démonstration publique de la connaissance de l'individu ou du groupe. C'est cette reconnaissance publique qui dote les individus des capacités d'expression et d'action sociales tant au plan individuel que collectif.

\section{En quête de visibilité. Le processus de la reconnaissance vu de l'intérieur}

\subsection{La visibilité en profondeur: ces formes de manifestation}

Une fois la visibilité définie comme une étape incluse dans le processus de la reconnaissance, nous allons nous plonger à l'intérieur de ce processus, en esquissant le portrait de chacune des phases dans lesquelles nous pouvons le fragmenter. La première des phases que nous allons analyser est celle de la visibilité.

Pour nous rapprocher de l'étude de la visibilité, il faut faire une remarque préalable. Celle-ci est une notion complexe qui peut se manifester sous plusieurs formes: matérielle, institutionnelle, sociale et médiatique. Nous allons insister ici sur l'étude des deux dernières, puisqu'elles ont, comme on le verra plus tard, de fortes implications dans l'évolution des processus migratoires.

a) La visibilité sociale: Lorsque nous parlons de la visibilité sociale, nous faisons référence à la capacité que possède un acteur d'être perceptible au niveau social. Cette capacité lui permet de s'insérer dans un système d'interaction complexe et de se manifester comme un acteur à part entière aux yeux des autres. En fait, il ne s'agit pas simplement de se percevoir soi-même comme 
sujet, mais de le faire à travers l'image que les autres projettent de soi. O. Voirol (2005: 16) donne, lui aussi, une autre définition de la visibilité sociale, la considérant comme «un processus par lequel des groupes sociaux bénéficient ou non d'une attention publique».

C'est pour cela, que le manque de visibilité dans les sociétés actuelles s'est transformé en un des moteurs déclencheurs de conflits sociaux et une des principales revendications de nombreux mouvements sociaux. Toutefois, il faut souligner que cette lutte est un chemin ardu et que parfois sa conquête n'est qu'éphémère, puisqu'elle se trouve assujettie à de nombreuses variables. Il n'est donc pas étrange qu'un groupe qui par plusieurs moyens est parvenu à avoir une certaine visibilité sociale puisse, à un moment donné, retomber de nouveau dans l'invisibilité. Retourner au point de départ peut être le produit de plusieurs circonstances, par exemple que le groupe n'ait pas la force suffisante pour attirer l'attention permanente de la société ou encore, que les médias, la société ou les politiciens ne le considère plus comme sujet d'intérêt. De ce fait, l'acquisition d'une visibilité sociale doit être toujours liée aux inquiétudes, intérêts, modes et va-et-vient qui traversent les sociétés.

b) La visibilité médiatique: Nombreuses ont été les études, dans le XXème siècle, qui ont été dédié aux effets et à l'influence des médias dans la transformation des imaginaires collectifs des sociétés, ainsi qu’à la révolution produite par leur nouvelle conception des relations spatio-temporelles. Comme souligne John B. Thompson (2000), la visibilité s'est vue aussi concernée par ces changements, du fait qu'elle ne peut plus être conçue seulement dans son sens d'expérience immédiate, régie par des relations de face-à-face et liée à la coprésence dans un même espace.

Le changement introduit par les médias se voit aussi accompagné par un autre élément lié à la visibilité. Leur capacité de relier des espaces très éloignés, en connectant un ensemble de plus en plus vaste de population, leur a conféré la faculté d'intervenir dans la formation de l'opinion publique et dans la construction des réalités discursives. Cependant la visibilité médiatique se caractérise également par son caractère incontrôlable, ce qui peut créer des effets positifs ou négatifs à n'importe quel groupe qui cherche la visibilité sociale par le biais des médias.

Nous pouvons présenter les médias comme les principaux représentants et diffuseurs des discours dominant dans les sociétés contemporaines. Néanmoins, nous considérons que sur le plan médiatique il faudrait tenir compte de l'existence d'autres supports qui, bien que minoritaires, sont le terrain de construction des contre-discours, et donc d'une interprétation différente de la réalité. Ces supports médiatiques alternatifs essaient de montrer, voire de rendre visible, ce qui est déformé ou même occulté par les médias de masse. La scène médiatique doit donc être conçue comme un espace qui ne manque pas de fortes dynamiques conflictuelles et de tensions entre différents discours (E. Macé, 2006). 
c) Visibilité vs invisibilité: Il faut considérer également que la visibilité peut se manifester non seulement sous des formes différentes, mais aussi sous divers degrés. Autrement dit, la visibilité peut être envisagée dans une échelle allant de l'invisibilité totale jusqu'à la pleine visibilité. Bien que chaque groupe d'individus se place différemment dans cette échelle de visibilité leur emplacement n'est pas toujours statique. Selon la trajectoire du groupe, son niveau d'organisation ou l'intérêt que sa situation suscite, son positionnement pourrait se modifier indistinctement vers chacun des deux pôles de cette échelle.

Il faut souligner également que l'expérience de l'invisibilité ainsi que celle de la visibilité n'implique pas à priori qu'elles aient respectivement une connotation négative ou positive, puisque toutes les deux peuvent se présenter comme un fait cherché par le groupe ou bien imposé par la société. Pour mieux illustrer ces différentes formes et degrés sous lesquelles la visibilité et l'invisibilité peuvent se manifester, nous allons nous centrer dans l'analyse de trois catégories de sujets :

— Les détenus. Cette première catégorie présente une visibilité particulière. La situation des prisonniers est paradoxale, puisque leur isolement du monde extérieur a fait que leur invisibilité physique est substituée par une forte visibilité symbolique due à la médiatisation de leur stigmatisation. Ce qui est intéressant dans l'observation de ce groupe, c'est que la visibilité et l'invisibilité rentrent singulièrement en collusion, exerçant deux actions parallèles de sanction. D’une part, le renforcement évident de leur distanciation physique et, d'autre part, l'affermissement de leur visibilité symbolique, consolidée par leur stigmatisation dans l'imaginaire sociale et utilisée, à la fois, pour faire accepter aux autres l'ordre établi².

- Les sans-abri. Nous définissons leur situation comme une invisibilité fictive, voire irréelle, puisque cette invisibilité a été construite à partir de la dénégation et du rejet que la société presque dans son ensemble exprime face à eux. En réalité, ils se trouvent à mi-chemin entre une forte visibilité ou perceptibilité physique et une invisibilité sociale presque absolue. Cependant leur situation est plus complexe, puisque leur «excessive» perceptibilité physique est considérée dérangeante et, par conséquent, elle est attaquée avec des mécanismes complexes qui visent à leur disparition au niveau social et à leur "fantômisation» au niveau physique.

- Les immigrés. Ce troisième groupe a une relation extrêmement complexe avec la visibilité. L'important ici n'est pas de discerner s'ils ont plus ou moins de visibilité, mais plutôt d'analyser comment ils sont représentés et quels sont les mécanismes qui provoquent cette visibilité. De ce point de vue leur visibilité va dépendre largement des facteurs tant externes qu'internes aux propres immigrés. Autrement dit, leur visibilité peut représenter un

2. Pour en savoir plus sur cette question consulter l'ouvrage de Michel Foucault (1975) où cet auteur développe l'argument sur la transformation de la visibilité en nouvel instrument du contrôle exercé par le pouvoir. 
fait souhaité ou bien subi ; la visibilité/invisibilité peut avoir des connotations négatives ou positives selon le contexte, les objectifs de l'immigré ou les discours dominants qui, sur eux, sont développés par les médias ou les structures institutionnelles d'un pays.

\subsection{L'incorporation d'une nouvelle étape: la "visibilisation"}

Il faut s'attendre à une réaction de la part des groupes lorsque ils subissent un manque évident de visibilité, lorsqu'ils ne se sentent pas insérés dans l'ensemble des relations et des interactions qui caractérisent toute société, ou, tout simplement, lorsqu'ils sont dans l'attente d'une visibilité sociale qui n'arrive pas à s'accomplir. Les attentes frustrées d'une visibilité sociale provoquent des réactions différentes. D’après O. Voirol (2005: 30), les individus ou les groupes réagissent au manque de visibilité de deux manières distinctes. D'une part, l'individu peut changer la perception qu'il a de lui-même, de son collectif et de sa place dans la société. Ce changement peut l'amener vers une situation de repli sur soi lorsque l'individu n'est pas capable de réagir face au discours dominant qui le transforme en déviant. D’autre part, le manque de visibilité sociale peut conduire à la formation d'un sentiment de révolte, de prise de conscience de soi-même ou de son collectif comme victime d'une situation qui est considérée injuste, provoquant la constitution de mouvements de résistance et/ou d'action collective, considérés par ces individus comme le dernier recours dans leur lutte pour devenir visibles.

Ainsi, la possibilité d'intervention du groupe exclu dans sa propre mise en visibilité ou l'intervention de certains agents externes au groupe dans cette démarche, doit être incorporée comme une nouvelle étape du processus de la reconnaissance. Nous allons nommer à cette nouvelle étape «visibilisation" et elle doit être placée entre l'invisibilité et la visibilité, donnant le schéma suivant: invisibilité-visibilisation-visibilité. Nous allons utiliser la notion de «visibilisation» avec une connotation particulière qui prend en partie un sens semblable à ce que O. Voirol (2005: 30) appelle «les opérations de constitution de la visibilité» et qu’il définit comme «l'ensemble des procédés par lesquels les auteurs se rendent visibles et viennent à exister pour autrui et pour leur environnement social». Pourtant, il faut insister sur le fait que l'étape de la visibilisation n'est pas le produit de l'action exclusive du groupe affecté d'invisibilité, mais aussi, et souvent de manière très déterminante, elle est induite par l'intervention d'autres acteurs extérieurs au groupe concerné.

Cette acception de la «visibilisation» trouve une place importante dans les travaux réalisés par l'auteur français Eric Macé (2006) et sa sociologie postcritique des média-cultures. D’après Macé (fortement influencé, entre autres, par le courant de pensée de Cultural Studies et les travaux d'Edgar Morin (1962)) le véritable enjeu du débat actuel sur la présence et la représentation des minorités dans les médias, se trouve dans le nécessaire dépassement du concept de visibilité et sa substitution par l'analyse sur la signification des processus de (in)visibilisation. Ce qui est intéressant pour cet auteur n'est pas de savoir «qui 
est montré» (fait qui nous renvoie à la notion de visibilité), mais plutôt d'analyser "comment les sujets sont montrés» (ce qui suppose se centrer sur les processus de (in)visibilisation). D'après Macé, l'emplacement depuis cette perspective permet de repérer avec plus de clarté l'existence des relations asymétriques de pouvoir entre les différents groupes qui constituent une société. Elle nous invite également à approfondir sur les causes qui sont derrière la construction des imaginaires $d$ ' "uautrui» dans les sociétés contemporaines, ainsi qu'à la mise en question des processus d'invisibilisation de la norme «universelle» masculine et blanche et la «sur-visibilisation» ou la «sous-visibilisation» des déviants à cette norme.

\subsection{Le dernier stade: la reconnaissance}

D'après A. Honneth (2005), la situation idéale, pour l'individu ou le groupe exclu, serait la possibilité de franchir avec succès chacune des phases décrites jusqu'à arriver à la reconnaissance de leur existence, de leur identité par autrui en tant que sujet ou en tant que groupe avec des caractéristiques spécifiques. De ce point de vue, la demande de la reconnaissance se transforme souvent en lutte. Une lutte qui se concentre dans le domaine social et institutionnel et qui a comme but ultime l'acquisition de l'estime de soi au travers de la reconnaissance par autrui. Le sujet jouissant de reconnaissance est celui qui, en faisant partie d'un corps social, bénéfice de tous les droits qui lui donnent accès à la pleine citoyenneté dans une société ouverte et caractérisée par le respect des différences (C. Taylor, 1992).

A. Honneth (2000) a particulièrement contribué à l'élaboration d'une théorie sur la lutte pour la reconnaissance à partir de la réactualisation des travaux d'Hegel. En fait, la reprise des théories hégéliennes lui a donné la possibilité de réédifier une sociologie de la naissance des mouvements sociaux à partir de l'expérience morale du mépris. Cette construction analytique permet à $A$. Honneth (2000: 193-195) de suggérer que l'irruption des mouvements de résistance et de protestation n'est pas simplement la conséquence de l'inégale répartition des moyens de subsistance entre les individus, mais que ces mouvements ont une logique morale résultant des attentes de reconnaissance fortement enracinées mais ignorées par la société. Ainsi, les mouvements d'action collective peuvent prendre consistance seulement si les individus qui ont souffert de l'expérience dramatique du mépris sortent de leur isolement et commencent à penser en tant que groupe.

La notion de reconnaissance telle qu'elle a été développée par A. Honneth ou C. Taylor a été largement contestée par un ensemble d'auteurs nord-américains, dont l'un des plus renommés est Nancy Fraser. Cette auteur développe une conception de la reconnaissance qui dépasse le multiculturalisme compatible avec les droits humains de C. Taylor (1992), ainsi que le déni de la reconnaissance comme déni moral d'A. Honneth (2000). N. Fraser (2005: 50) affirme la nécessité de traiter la reconnaissance comme une question de justice, ce qui permet de «faire du déni de la reconnaissance un tort relevant du 
statut, situé dans les relations sociales, et non dans la psychologie». Elle s'oppose à la formulation de la reconnaissance en tant qu'élément indiscutable de la formation de l'identité individuelle, la considérant génératrice des dynamiques sectaires. Pour surmonter cette problématique, Fraser (2005: 80-81) propose un autre type de modèle centré sur le statut social, où elle définit le déni de reconnaissance comme une relation institutionnalisée de subordination sociale qui empêche la participation à part entière de certains sujets ou groupes dans les interactions sociales qui se développent au sein d'une société.

Dans notre analyse cette dernière étape de la reconnaissance représente l'achèvement du processus dans son ensemble. Ainsi, chacune des phases décrites peuvent être extrapolées à un schéma explicatif présentant une structure complexe, dynamique, non unidirectionnelle et marquée par de fortes tensions entre les différents acteurs qui interviennent dans le processus. Ce schéma aurait donc la structure suivante:

\section{Invisibilité - (In) Visibilisation - Visibilité — Reconnaissance.}

Présenter le schéma sous cette forme permet d'accentuer l'existence, tout au long du processus, de dynamiques conflictuelles qui engendrent que celui-ci ne se présente jamais sous une forme monodirectionnelle. Sans perdre de vue les logiques de domination présentes au sein des sociétés, qui sont derrière tout déni de reconnaissance, il faudrait prendre en compte qu'elles rencontrent toujours la résistance et la contestation des groupes affectés par l'invisibilité ou le déni de reconnaissance. Nous ne pouvons penser les relations sociales qu’à partir de cette conflictualité continuelle qui dynamise les espaces publics et qui explique les moments d'évolution et de blocage qui traversent les conflits sociaux.

C'est à partir de cette perspective que nous allons centrer notre étude sur la figure de l'immigré, en portant une attention particulière à son positionnement par rapport à la visibilité, ainsi qu'aux processus de (in)visibilisation qui vont déterminer inévitablement sa place dans l'imaginaire des sociétés d'installation.

\section{La visibilité et ses implications dans la migration}

On ne peut pas parler actuellement d'une théorie qui puisse expliquer les phénomènes migratoires dans sa totalité, mais de l'existence de plusieurs cadres théoriques qui n'offrent que des explications partielles aux migrations. Néanmoins, pour clarifier notre démarche, nous allons utiliser comme cadre de référence les théories historico-structurelles, c'est-à-dire celles qui tiennent compte des transformations qui ont lieu à l'intérieur des sociétés de réception. Plus concrètement nous allons nous appuyer sur le modèle théorique des réseaux sociaux, en utilisant la visibilité comme un outil analytique complémentaire aux explications apportées par ce modèle. La théorie sur les réseaux sociaux insiste sur le fait que les flux migratoires sont un produit et non seulement 
social mais aussi économique. Les réseaux se constituent à partir de relations interpersonnelles qui connectent trois types d'espaces: les lieux d'origine, les lieux d'installation et des espaces flous qu'on dénomme "transnationaux». En outre, la théorie des réseaux renforce la vision des processus migratoires comme un système des flux directement conditionnés par les structures sociales et politiques indissociables aux histoires des sociétés d'émission et de réception des migrations (Ubaldo Martínez Veiga, 2004). En ce sens, ce modèle théorique insiste sur l'existence d'une série de facteurs qui influencent directement le déroulement des processus migratoires. Ces facteurs (politiques des gouvernements récepteurs d'immigration, conditions du marché du travail, caractéristiques des communautés éthniques, puissance et effectivité des réseaux communautaires, opinion publique sur l'immigration etc.) n'affectent pas exclusivement l'insertion des immigrés dans le marché du travail, mais aussi leur insertion sociale, leur interaction avec la société d'accueil et en définitive leur perceptibilité en tant qu'acteurs sociaux. C'est ici que l'analyse en terme de visibilité devient importante. Ce nouvel instrument permet de compléter le modèle, en attirant notre attention sur les mécanismes qui contribuent à la formation des imaginaires collectifs sur l'immigration dans les sociétés de réception, et en conditionnant énormément les comportements des immigrés dans leur installation dans un pays. Dans ce sens, l'étude sur la visibilité tient compte d'une double problématique. D'abord, comment, pourquoi et à quels moments les immigrés sont montrés; ce qui nous amène à analyser la formation des discours d'inclusion/exclusion développés au sein des sociétés de réception. En suite, on peut constater que les réseaux migratoires développent, entre autres fonctions, celles de l'appui et de la protection aux immigrés, afin de contrecarrer les effets non désirés de l'installation dans des contextes marqués par l'hostilité à leur présence. Ces réseaux les accordent, en même temps, la possibilité d'agir et d'influencer leur propre mise en visibilité dans la sphère publique des sociétés d'installation.

Nous allons analyser, par la suite, la relation complexe existante entre migration et visibilité à partir du cadre théorique énoncé précédemment. Pour y parvenir, nous allons utiliser comme terrain d'application la situation et la représentation des immigrés en Espagne. Certes, l'utilisation du contexte migratoire espagnol ne peut pas être comprise ici comme le résultat d'un travail de terrain exhaustif, mais elle nous a servi justement pour clarifier notre démarche théorique. En outre, il faut souligner que la véritable application du cadre théorique présenté dans cet article correspond à l'étude particulière et approfondie sur deux migrations en Espagne, Kurdes et Sahraouises, dont les résultats seront publiés prochainement.

\subsection{Le processus de mise en visibilité}

Notre analyse de la relation visibilité/migration tourne au tour de l'hypothèse que le couple visibilité/invisibilité chez les immigrés est déterminé par deux types de composantes: endogènes et exogènes. Ces éléments se trouvent à l'in- 
térieur de ce que nous avons appelé le processus de "visibilisation» et ils doivent être toujours pensés en combinaison. La plus forte influence d'un facteur ou d'un autre dans le processus de mise en visibilité va dépendre largement d'autres variables comme par exemple: la tradition migratoire du pays d'accueil, les origines de l'immigré, l'existence d'éléments historiques, géographiques ou culturels qui lient le pays d'origine de l'immigré et le pays de réception, etc.

Dans le cas de l'Espagne, le passage rapide de l'émigration vers l'immigration a eu des conséquences importantes dans la perception sur la présence des immigrés dans la société espagnole. La croissance économique flamboyante du pays a provoqué un décalage entre l'idée de modernité, représenté par l'essor économique, et l'idée de sub-développement pensé comme intrinsèque à la figure de l'immigré. En outre, les liens historiques et géographiques ont joué aussi un rôle important, non seulement dans l'entrée plus poussée de certaines populations provenant du continent Sud-Américain ou de l'Afrique du Nord, mais aussi, dans la reproduction de certaines images autour de ces communautés d'immigrés.

a) Facteurs endogènes: Lorsque nous parlons de facteurs endogènes, nous faisons référence à ceux qui sont déterminés par la propre trajectoire migratoire d'un sujet ou d'un groupe. Ce parcours peut être défini depuis le début de la migration ou souffrir de modifications considérables selon les obstacles rencontrés pendant le voyage, selon les caractéristiques du contexte d'installation ou l'efficacité du réseau immigrant mis en place. De ce fait, selon l'étape du processus migratoire chaque communauté d'immigrés et, même chaque sujet, se positionneront de façon différente par rapport à la visibilité. Nous avons distingué l'existence de trois phases dans l'installation des immigrés dans un pays $^{3}$, correspondant aussi à trois formes différentes d'utilisation de la visibilité par ceux-ci ${ }^{4}$ :

1. Phase de transition: Cette première étape comprend la période qui va dès la sortie de la région d'origine jusqu'à l'entrée dans le pays d'installation. Elle peut être plus ou moins longue et dépend du voyage (direct ou en plusieurs escales), des routes choisies et des obstacles trouvés. Il est aussi pos-

3. Il faut clarifier le fait que les étapes que nous proposons du processus migratoire ne peuvent pas être pensées comme un prototype fermé (avec un début et une fin), unique et uniformisant. Nous sommes conscients, bien au contraire, qu'une des caractéristiques les plus importantes des mouvements de populations contemporains est leur plasticité et leur hétérogénéité. Cela veut dire, tout simplement, que la migration n'est pas régie par des facteurs constants, mais par une série de variables qui modifient toujours le processus.

4. Les différentes étapes du processus d'installation des immigrés que nous proposons ici, est une interprétation particulière du schéma élaboré par l'auteur catalan Jordi Moreras (1999: 334-335) sur les différentes étapes qui composent la formation de la communauté d'immigrants musulmans à Barcelone. Cet auteur distingue fondamentalement trois étapes ou phases qu'il dénomme: 1 . Phase d'abandon temporaire. 2. Phase de récupération ou rencontre et 3. Phase de réaffirmation et stabilisation. 
sible que les migrants n'arrivent jamais à rentrer dans le pays choisi, restant dans des zones intermédiaires, ou bien qu'une fois dans le pays de préférence, celui-ci se transforme encore en endroit de passage vers un autre pays. Cette première phase se caractérise par l'ambigüité et, en générale, par la recherche d'une invisibilité, permettant à celle-ci la fluidité des mouvements.

2. Phase d'installation: La situation de l'immigré par rapport à la visibilité peut à être assez ambiguë dans cette deuxième étape, car elle dépend largement de son encadrement dans un secteur concret du marché du travail et de l'accomplissement de son processus de régularisation. La réussite, ou non, de ces deux facteurs combinés, conditionnera leur décision de rester dans l'anonymat, ou bien, de s'engager dans la recherche d'une visibilité sociale plus forte. Cet engagement a lieu, couramment, à l'intérieur d'un cadre communautaire d'appartenance. L'existence des réseaux communautaires joue donc un rôle de premier ordre dans l'insertion des nouveaux arrivés dans la société de réception. Ces réseaux, constitués à partir de la solidarité inter-groupale, fournissent aux immigrés une protection, face à un environnement étrange et parfois hostile à leur présence, en leur procurant certains services qu'ils ne peuvent pas obtenir par eux-mêmes. Ils agissent, en même temps, en fonction des caractéristiques propres à chaque communauté d'immigrés. La protection exercée par le réseau communautaire permet aussi l'extériorisation de diverses pratiques culturelles qui accélèrent énormément le processus de mise en visibilité. Par exemple, ce fait est extrêmement perceptible au sein des communautés musulmanes installées en Espagne. Leurs actions de groupe leur ont permis entre autres choses, d'ouvrir un dialogue avec les administrations de l'Etat espagnol et de présenter leurs revendications, tant identitaire que de participation égalitaire au sein de la société espagnole.

3. La stabilisation: En principe, cette troisième étape suppose l'achèvement du processus migratoire, c'est-à-dire que l'immigré parvient ici à une stabilité ou une normalisation économique et juridique, et il souhaite que cette stabilité se reflète aussi dans d'autres domaines de sa vie, notamment en ce qui concerne sa parité de participation en tant que membre actif d'une société. Ce qui caractérise cette phase de stabilisation est le passage, toujours dans le cadre d'un réseau organisé, de la visibilité vers la lutte pour la reconnaissance de leurs droits sociaux et institutionnels.

Nous sommes conscients de l'impossibilité d'établir un modèle de comportement généralisé, pour l'ensemble des populations immigrées en Espagne, en ce qui concerne la forme qu'elles ont de gérer leur visibilité dans chacune de ces phases. Ce que nous proposons ici, c'est la construction d'un instrument d'analyse, nous permettant d'étudier le comportement de certaines communautés d'immigrés dans chacune des étapes décrites précédemment par rapport à la visibilité et le rôle que jouent sur ce sujet les réseaux communautaires. Ainsi, afin d'avancer quelques données de la recherche que nous sommes en 
train d'effectuer, nous allons prendre comme exemple pratique la communauté kurde et sahraouie en Espagne. Nous pouvons constater que ces deux petites communautés développent deux formes complètement différentes de gestion de leur visibilité dans les trois phases décrites précédemment. Bien que les problèmes politico-nationaux dans leurs régions d'origine soient à la base de leur migration, la conception de celle-ci est bien différente dans chacun des deux groupes. Leur interaction avec le contexte migratoire espagnol joue aussi un rôle important dans la façon de construire leurs réseaux communautaires, de concevoir leurs relations sociales, ou encore, de poser leurs revendications de visibilité dans la phase d'installation et de stabilisation. Ainsi, nous pouvons avancer que la faible présence des Kurdes en Espagne, jointe à leur assimilation à d'autres populations immigrées provenant du Moyen Orient, a renforcé leur statut d'invisibles dans le pays. Ils ont seulement pu contrecarrer ce fait une fois que, dans la phase de stabilisation, ils ont eu la possibilité d'investir leurs efforts dans la formation d'un réseau économique large et basé sur des relations de solidarité autour de l'entreprise ethnique du Kebab. Celui-ci devenant le milieu de visibilité et d'intermédiation, dans l'espace publique, entre les Kurdes et la société espagnole. En ce qui concerne les Sahraouis, leur visibilité, tant dans la phase d'installation que dans la phase de stabilisation, est beaucoup plus forte grâce à l'inexorable connexion entre le conflit du Sahara Occidental et l'Etat espagnol. Leurs stratégies par rapport à l'acquisition d'une plus forte visibilité ont privilégié, notamment, la formation d'un réseau associatif, capable de leurs procurer de l'appui et de la protection, particulièrement dans las deux premières étapes de leur séjour en Espagne. Mais surtout, ils ont fait usage du réseau associatif pro-sahraoui, déjà existant dans le pays et constitué par des espagnols, pour pousser leurs revendications et faire connaître leur cause à un nombre plus large de personnes.

Comme ont verra par la suite, la prise de visibilité, voulue ou subie, de certaines communautés d'immigrés dans le pays a été marquée par un environnement d'hostilité ou, au moins, de méfiance. La société espagnole, en générale, a eu du mal à interpréter certains signes externes liés à leur culture et à admettre leur irruption de plus en plus grandissante dans l'espace public. L'appropriation de certains milieux du domaine public a été considérée par une partie de la population comme l'usurpation, voire l'invasion intolérable d'un espace qui leur appartient ${ }^{5}$. Dans ce contexte la visibilité sociale peut se

5. Dans presque l'ensemble des sociétés d'installation, le manque d'une vraie reconnaissance des immigrés en tant qu'acteurs sociaux va de paire avec les difficultés d'expression rencontrées par ceux-ci dans l'usage de l'espace public. A travers le mécanisme du déni de reconnaissance, la structure institutionnelle d'un pays et sa propre société peut essayer de contrôler, voire de limiter l'utilisation de l'espace public qui appartient au cadre national, contestant l'usage de celui-ci par ceux qui ne sont pas considérés des nationaux ou, tout simplement, pour ceux qui n'accomplissent pas les conditions nécessaires pour y accéder. Cependant, comme le souligne E. Macé (2006) l'espace public, en étant asymétrique et traversé par des rapports de pouvoirs et de domination, est caractérisé aussi par l'existence de fortes tensions et dynamiques conflictuelles. Traiter l'espace public comme une sphère 
transformer pour l'immigré en fardeau, puisque il n'est plus capable de gérer les perceptions de sa propre image, voire de contrôler la manière dont il est perçu. La lutte des immigrés dans le domaine de la visibilité, dans un pays qui a encore du mal à s'accommoder au phénomène migratoire, devient donc double. D'une part, ils essayent de se rendre visibles en tant que sujets sociaux qui contribuent au développement économique et social du pays. D'autre part, ils doivent lutter pour déconstruire les images, souvent négatives, fausses ou déformées, que les institutions et la société ont crée d'eux.

b) Facteurs exogènes: Comme nous l'avons indiqué plus haut, la visibilité est un processus complexe dans lequel intervient une multiplicité de facteurs qui sont aussi extérieurs aux propres collectifs d'immigrés. Dans les dernières décennies, l'immigration s'est transformée en Espagne en sujet d'intérêt ainsi que d'inquiétude. Cela s'est considérablement manifesté au travers de sa présence de plus en plus évidente dans les médias, dans le domaine des sciences sociales et dans les débats politiques.

L'intervention de certains acteurs (politiciens, médias, opinion publique, scientifiques sociaux) dans la visibilisation des populations immigrées n'a pas toujours contribué à la construction d'une image positive de l'immigré dans le pays. Bien au contraire, cette image a été souvent bâtie à partir de préconceptions éloignées de la réalité, pleines de connotations négatives et de préjugés. L'intervention de ces acteurs a rendu visible l' «autre», mais toujours dans son "étrangeté», comme une présence supportée en tant que nécessaire au niveau économique, mais rejetée au niveau social. De ce fait, ils ont contribué à la radicalisation de l'altérité, transformant celle-ci en quelque chose de négatif, car la présence de l'autre est vue, la plupart des fois, comme une menace pour l'ordre social. Comme le remarque l'auteur espagnol Enrique Santamaria (2002), l'immigré n'est plus regardé en tant que sujet social, contribuant au développement positif de la société de réception, mais il est plutôt perçu et décrit par le discours dominant comme un grave problème qui affecte systématiquement toutes les sociétés d'installation.

Les positionnements des gouvernements espagnols successifs et les moyens de communications, ont joué un rôle essentiel dans la construction des imaginaires collectifs et des discours homogénéisants sur la catégorie de l'immigré. En particulier les médias se sont transformés en puissants agents de visi-

marquée par la conflictualité implique de prendre en compte non seulement des discours des acteurs hégémoniques mais aussi d'introduire d'autres acteurs et d'autres discours. En outre, il faudrait considérer la sphère publique comme un espace aux contours flous, où il existe plusieurs scènes (que N. Fraser (2005) appelle "contre-publics subalternes») qui se développent particulièrement au niveau local et qui essaient d'accéder, et de faire parvenir leurs discours, à une sphère publique plus large, nationale ou globale. Ainsi, E. Macé (2006: 33) signale que «c'est cette diversité de points de vue qui fait finalement de la sphère publique un espace plastique au sens où ses contours ne sont pas prédéterminés mais varient à mesure que de nouveaux problèmes ou de nouveaux acteurs apparaissent et demandent à être pris en compte». 
bilisation. Les discours qu'ils articulent par rapport à l'immigration ont fait de l' «autre» un être omniprésent. Pourtant, cette omniprésence a provoqué paradoxalement son invisibilité comme sujet individuel. La catégorie abstraite, agglutinante et socialement construite de l'«immigré» a été objet d'une telle médiatisation que sa présence est devenue finalement trop perceptible, au point de déranger.

Par rapport à ce sujet les médias articulent souvent un double discours de visibilisation/invisibilisation qui suit des logiques en principe contradictoires mais qui, en réalité, se complétent parfaitement. Ainsi, par exemple, nous pouvons très bien discerner ce double discours à partir de l'analyse de deux événements qui ont eu lieu en Espagne au début du XXIème siècle.

D'une part, le cas des émeutes du village El Ejido ${ }^{6}$ dans la province d'Almeria au sud de l'Espagne dans l'année 2000 constitue un exemple paradigmatique d'invisibilisation de la population immigrante. En effet, les événements déclencheurs des émeutes contre la population immigrée dans cette région du pays cachaient derrière une politique très concrète d'occultation des conditions de vie extrêmes des travailleurs immigrés. Cette situation poussée par les entrepreneurs agricoles de la zone était bien connue des administrations publiques et des moyens de communication. Ce fait facilitait principalement deux choses. D'un côté, la réification de la figure de l'immigré, justifiant ainsi son exploitation sans frein et l'acceptation de la situation par la population d'El Ejido. D'un autre coté, le fait de rendre invisible ces pratiques évitait la formation d'une opinion publique pouvant être contraire à cette forme d'exploitation et donc contraire aux intérêts économiques des entrepreneurs de la région.

Le second de nos exemples est plus récent. Il montre les effets sur la population espagnole qu'a eu la «sur-visibilisation» des immigrés arrivant dans les côtes des Îles Canaries en 2006. L'été et l'automne 2006 se sont incontestablement caractérisés par l'augmentation du nombre d'immigrés arrivés par voie maritime en Espagne. Nous constatons également une diversification des routes d'accès, celles-ci s'étant déplacées vers les Îles Canaries et la côte plus orientale de l'Andalousie. Malgré cela, les discours officiels ont surtout fait attention à l'accroissement du nombre d'arrivés plutôt qu'aux véritables causes qui expliquaient comment et pourquoi le phénomène s'était déclenché. La présence continue - à la télévision, à la radio et dans la presse- des nouvelles relatives à l'entrée d'immigrés dans le pays par voie maritime, a eu comme résultat la création d'une alarme sociale sans précédent dans l'opinion publique espagnole. Si nous faisons un parcours détaillé des nouvelles apparues sur le sujet dans la presse écrite à cette époque, nous pouvons remarquer l'influence qu'a eue l'utilisation constante de certains mots dans le renforcement de la panique sociale.

Dans les deux cas analysés, les stratégies d'occultation et de visibilisation déployées par le discours dominant, officiel et médiatique, obéissent à une

6. Pour avoir une vision plus approfondie sur les conditions sociales et économiques qui entourent la problématique du secteur agricole dans cette municipalité de la province d'Almeria, voir U. Martínez Veiga (2004). 
seule et unique logique visant le discrédit de l'immigré. Celui-ci se trouve ainsi toujours attrapé dans une double situation qui le rend, dans certains cas, trop visible en tant que différent, en tant qu' "autre», et, dans d'autres cas, tout simplement inexistant.

\subsection{Les marqueurs de visibilité en migration}

Nous allons finalement exposer le processus qu'on a suivi pour l'étude de la visibilité dans le cas de l'immigration des Kurdes et Sahraouis en Espagne. En attendant la publication des résultats de cette recherche, nous allons présenter ici les traits généraux d'analyse utilisés, en prenant le collectif d'immigrés dans son ensemble dans le contexte migratoire espagnol. L'analyse des migrations à partir de la théorie des réseaux nous amène à pencher notre regard sur les contextes où se déroulent les processus de visibilisation et à comprendre pourquoi certaines communautés sont plus perceptibles que d'autres dans un pays. Ainsi, pour l'étude approfondie de la visibilité dans un contexte concret, nous avons utilisé plusieurs paramètres combinés qu'on a dénommés «marqueurs de visibilité». Ceux-ci déterminent quelles sont les conditions qui accompagnent le processus de visibilisation, ainsi que le degré et la forme de visibilité que présente un collectif d'immigrés déterminé. Certes, nous pourrions formuler plusieurs combinaisons ou ajouter d'autres paramètres à la liste, mais nous croyons que ceux-ci sont les plus importants pour l'étude de la visibilité appliquée aux phénomènes migratoires:

1. Traitement que font les moyens de communication officiels de l'immigration, tant au sens qualitatif que quantitatif.

2. Analyse de la mise en visibilité de ces populations par les statistiques officielles. Elles nous montrent la contribution de l'immigration à l'évolution démographique et économique du pays et l'impact produit par la publication de celles-ci au sein d'une société espagnole.

3. Problématisation de l'immigration dans le discours politique publique: parti politiques, syndicats, mouvement sociaux, organisations non gouvernementales, associations d'immigrés etc.

4. Le marché du travail peut être aussi utilisé comme marqueur de visibilité. La structure du marché de travail espagnol détermine fortement la place occupée par les immigrés dans la société, ses relations sociales, ainsi que le type de discours qu'une partie de la population construit autour d'eux.

5. Etude des lois et des politiques publiques élaborées par l'Etat espagnol en ce qui concerne la migration. Leur analyse peut donner une image claire de l'importance que les gouvernements successifs ont accordé au sujet de la migration et de l'état du débat public sur la question, générant des discours pour et contre l'immigration.

6. Le nombre et l'importance des associations d'immigrés, ainsi que les réseaux sociaux dans lesquels ils s'appuient. Comme nous l'avons déjà signalé, l'acquisition de la visibilité sociale se manifeste également au travers de leur 
capacité de mobilisation collective. La structuration du groupe autour d'une organisation plus ou moins puissante va être déterminante pour faire parvenir leurs discours et leurs revendications vers l'espace public.

7. Etude sur la localisation spatiale des immigrés, et notamment des espaces urbains à partir de la configuration des réseaux sociaux. Dans leur perceptibilité sociale, un rôle capital est joué par la concentration ou la dispersion spatiale tant au niveau du logement qu'au niveau des emplacements des lieux de travail.

8. On peut aussi repérer leur capacité d'appropriation de l'espace public. C'est-à-dire la possibilité de se rassembler dans des endroits publics, de se regrouper dans certains quartiers, de constituer des endroits de culte et d'association, de développer des entreprises ethniques, d'être présents dans certains établissements publics, par exemple, leur présence dans les écoles. Toutes ces activités dénotent la présence de plus en plus incontestable des immigrés dans l'espace public (urbain) amplifiant leur visibilité.

9. Analyse de la place qu'occupe la migration dans la production scientifique en Espagne. L'examen des travaux de recherche, tant au sens qualitatif que quantitatif, est un marqueur de l'évolution dans le temps de l'immigration dans le pays et il a une influence notoire dans les processus de visibilisation des collectifs d'immigrés.

\section{Conclusion}

L'intention principale de ce texte a été de faire sortir la notion de visibilité de sa marginalité comme outil analytique en sciences sociales. De ce fait, nous sommes parvenus dans un premier temps à problématiser le terme de visibilité à partir de sa mise en relation avec le processus de la reconnaissance. Notre objectif a été d'apporter une nouvelle interprétation de ce processus, en le décortiquant en plusieurs étapes, dont la visibilité en ferait partie. L'étape de la visibilité revêt une importance capitale dans ce processus, car comme nous l'avons démontré, on ne peut pas aboutir à la reconnaissance sans avoir acquis auparavant une visibilité sociale. En définitif, cette démarche nous a permis d'accomplir un double objectif. D'abord, nous avons pu différencier la notion de visibilité et celle de reconnaissance, souvent, toutes les deux, objets d'une confusion conceptuelle. Ensuite, nous avons présenté le processus de la reconnaissance comme un processus non homogène, mais plutôt composé par l'assemblage de plusieurs actions et caractérisé par la conflictualité dans chacune des phases qui le constituent.

Une fois esquissés les traits fondamentaux de la notion de visibilité, nous avons appliqué cet outil d'analyse aux phénomènes migratoires, en nous appuyant sur le modèle théorique des réseaux sociaux. Nous avons démontré la pertinence d'utiliser la visibilité comme facteur complémentaire aux explications apportées par ce modèle au sujet des migrations, car ce facteur est redondant sur les aspects sociaux qui conditionnent et influencent l'installation des immigrés dans les sociétés de réception. La consolidation des réseaux 
migratoires contribue énormément à rendre visible la migration, en même temps qu'ils exercent un travail d'appui et de protection des nouveaux arrivés face à un environnement caractérisé, parfois, par l'hostilitè à leur présence. L'analyse sur la visibilité nous a permis d'expliquer, en définitive, quelles sont les conditions qui se trouvent derrière la production des discours d'inclusion/exclusion sur l'immigration, ainsi que la capacité des propres immigrés à gérer leur propre visibilité dans les sociétés de réception.

\section{Références bibliographiques}

Foucault, M. (1975). Surveiller et punir. Paris: NRF, Gallimard. Bibliothèque des Histoires.

Fraser, N. (2005). Qu'est-ce que la justice sociale? Reconnaissance et redistribution. Paris: Éditions La Découverte.

Ricoeur, P. (2004). Parcours de la reconnaissance. Trois études. Paris: Éditions Stock. Honneth, A. (2000). La lutte pour la reconnaissance. Paris: Éditions le Cerf.

- (2005). «L'invisibilité: sur l'épistémologie de la reconnaissance». Réseaux, vol. 23, $\mathrm{n}^{\mathrm{o}} 129-130$, p. 41-57.

MACÉ, E. (2006). Les imaginaires médiatiques. Une sociologie postcritique des médias. Paris: Amsterdam.

Martínez Veiga, U. (2004). Trabajadores invisibles: Precariedad, rotación y pobreza de la inmigración en España. Madrid: Catarata.

Moreras, J. (1999). Musulmanes en Barcelona. Espacios y dinámicas comunitarias. Barcelona: CIDOB.

SANTAMARIA, E. (2002). La incógnita del extraño: Una aproximación a la significación sociológica de la inmigración no comunitaria. Barcelona: Anthropos.

TAYLOR, CH. (1992). Multiculturalism and "the Politics of recognitions». Princeton: Princeton University Press.

Thompson, J. B. (2000). «Transformation de la visibilité». Réseau, 100, p. 189-213.

- (2005). «La nouvelle visibilité». Réseaux, vol. 23, no 129-130, p. 60-87.

VoIROL, O. (2003). «L'espace public et les luttes pour la reconnaissance. De Habermas à Honneth». En: Barril, C.; CARrel, M.; Guerrero J. C.; MárqueZ, A. (dir.). Le public en action, usages et limites de la notion d'espace public en sciences sociales. Paris: L'Harmattan, p. 117-137.

- (2005). «Visibilité et invisibilité: une introduction». Réseaux, vol. 23, no 129-130, p. 9-36.

- (2005). «Les luttes pour la visibilité. Esquisse d'une problématique». Réseaux, vol. 23, no 129-130, p. 91-118. 